\title{
Yannick Estienne, Le journalisme après Internet
}

Paris, Éd. L'Harmattan, 2008

\section{Amandine Degand}

\section{OpenEdition}

\section{Journals}

Édition électronique

URL : http://journals.openedition.org/questionsdecommunication/915

DOI : 10.4000/questionsdecommunication.915

ISSN : 2259-8901

\section{Éditeur}

Presses universitaires de Lorraine

\section{Édition imprimée}

Date de publication : 1 juillet 2009

Pagination : 443-445

ISBN : 978-2-86480-989-0

ISSN : $1633-5961$

\section{Référence électronique}

Amandine Degand, «Yannick Estienne, Le journalisme après Internet », Questions de communication [En ligne], 15 | 2009, mis en ligne le 17 janvier 2012, consulté le 22 septembre 2020. URL : http:// journals.openedition.org/questionsdecommunication/915 ; DOI : https://doi.org/10.4000/ questionsdecommunication.915

Ce document a été généré automatiquement le 22 septembre 2020

Tous droits réservés 


\title{
Yannick Estienne, Le journalisme après Internet
}

\author{
Paris, Éd. L'Harmattan, 2008
}

Amandine Degand

\section{RÉFÉRENCE}

Yannick Estienne, Le journalisme après Internet. Paris, Éd. L'Harmattan, coll. Communication et civilisation, 2008, $312 \mathrm{p}$.

1 C'est d'une manière quasi clinique que l'ouvrage de Yannick Estienne inventorie le domaine du journalisme web en France. Il ouvre la voie par les métiers « hors média » de l'information en ligne: éditeur, agencier, agrégateur ou encore entreprenaute. L'auteur s'intéresse également à la problématique des métiers de l'information et du journalisme web, dont les frontières sont encore plus floues que celles du journalisme en général. Face à l'hybridation des profils de journalistes web, la spécialité peine à se professionnaliser. Mais cette professionnalisation du journalisme web, aussi compliquée soit-elle, est-elle pour autant impossible? Pour Yannick Estienne, cela ne fait aucun doute : la spécialité n'aurait pas encore été « saisie collectivement » par ceux qui la constituent. Il n'y a pas eu en amont le travail politique nécessaire à la mobilisation de la spécialité en tant que groupe professionnel, et ses membres s'identifient dès lors davantage globalement à la profession journalistique, et à leur entreprise de presse en particulier.

2 Ce constat est le fruit d'une thèse de doctorat en sciences de l'information et de la communication, soutenue à l'université Stendhal-Grenoble 3 en 2006, au cours de laquelle Yannick Estienne, chercheur associé au Centre de recherches sur l'action politique en Europe de l'université Rennes I, s'est attelé à interviewer une centaine d'acteurs, noyau dur du microcosme social du journalisme web français. Les réponses de ceux-ci dénoncent souvent un certain désenchantement par rapport à leur profession, et parfois, des entorses à la déontologie. Telle est par exemple 
l'intervention d'un ex-journaliste web parisien qui avoue que, hormis pour le JT en ligne, il recevait des commandes de gros clients sur l'internet. « Il fallait orienter notre article d'une façon précise, suivant ce qu'ils voulaient, toujours rester positif » relate-til. Un autre professionnel constate avec effroi ce que veut dire journalisme de desk quand il déplore : «On est vissés à nos bureaux comme on ne l'a jamais été ».Quant au récit de la journée type d'une journaliste web du Progrès, il dévoile la réalité des pratiques. Entre copié-collé des articles de la veille, classement dans les rubriques ad hoc et veille de la mise en ligne automatique, la journaliste n'a même pas le temps de lire les articles qu'elle traite.

3 Anonymes qui travaillent dans l'ombre sans jamais signer leurs articles virtuels, les acteurs du journalisme online sont condamnés à une «double offense » : frustrés de ne pouvoir s'adonner à une écriture stylisée et créative, ils sont en outre forcés de mettre en ligne les articles de la prestigieuse rédaction papier, de valoriser les productions des autres. Professionnellement, ils n'existent que comme « auxiliaires de la machine » qui les emploie. Nous nous retrouvons donc face à un parent pauvre du journalisme, méprisé par ses pairs et accusé de vouloir « cannibaliser » son prédécesseur papier. De plus, d'après la définition de Julien Duval (Critique de la raison journalistique. Les transformations de la presse économique en France, Paris, Éd. Le Seuil, 2004, pp. 330-331) du capital journalistique, force est de constater que les journalistes web en sont peu revêtus. "Peu nombreux, invisibles et inconnus du public, ces derniers disposent de très peu de pouvoir [...] et effectuent un travail sinon ingrat, du moins peu valorisant " (p.139); un constat qui fait dire à Yannick Estienne que le journalisme web est " dominé ». Il est tout en bas dans la hiérarchie professionnelle.

Et pour alourdir encore le constat, l'auteur remarque que le métier, en proie à la multiplication des blogs, se voit banaliser. L'indifférenciation ne cesse de croître entre les journalismes professionnels et non-professionnels. Dans ce cas, le journaliste n'est plus que l'ombre de sa grandeur passée, celle de l'époque où il était l'un des seuls privilégiés à avoir accès aux sources, à pouvoir donner de la voix : «Les journalistes craignent par conséquent de perdre leur identité, leur légitimité et leur magistère " (p. 187). Malgré cette crise de légitimité du journalisme web, Yannick Estienne rappelle, dans son histoire récente de la presse en ligne, qu'elle n'est encore que le nouveau-né de l'information. Perçue à ses débuts comme "un jeu sans véritable enjeu», elle a beaucoup évolué. Son Minitel de prédécesseur fait déjà figure d'ancêtre. Au début des années 80 , l'introduction de la presse électronique a estompé les frontières entre les différents métiers journalistiques, de la rédaction à la fabrication. Avec l'introduction de la PAO (programmation assistée par ordinateur), le journaliste voit ses compétences se diversifier, et sa charge de travail croître. Et quand l'internet débarque, malgré les réticences, il faut adapter ses pratiques. La révolution web est entamée. Son idylle sera de courte durée. Dès 2001, une crise vient brutalement briser les ambitions des sites de presse et des start-up, trois ans à peine après le début de leur envolée. Entre-temps, l'information en ligne est pourtant devenue incontournable. Autant que les études qui en traitent.

5 Comme l'indique Érik Neveu, professeur à l'Institut d'études politiques de Rennes, dans sa préface de l'ouvrage, de nombreux constats de Yannick Estienne rencontrent ceux des chercheurs de l'université Laval à Québec : la prolifération des soft news et des infosservice, la difficulté croissante à différencier journalisme, information recyclée et communication, le succès d'un journalisme citoyen et le remodelage du journalisme 
pour le faire entrer dans le moule des logiques commerciales. Ce dernier point fait l'objet du sixième chapitre de l'ouvrage de Yannick Estienne qui considère le journalisme web comme soumis aux logiques de marketing. Il démontre notamment que les techniques de mesure d'audience n'ont jamais été aussi fines que sur le web. Elles répondent donc parfaitement aux logiques de marketing puisque elles pourraient orienter les choix éditoriaux. Le risque est dès lors éthique : le meilleur article est-il forcément celui qui récolte un maximum de clics? On constate en tout cas la recrudescence des soft news, des infos pratiques et des modules vidéo, photo et audio.

Dans ces perspectives peu encourageantes, on ne peut que s'inquiéter de l'avenir de la spécialité. Et c'est sans doute cette incertitude qui donne lieu aux thèses les plus intéressantes de Yannick Estienne qui s'interroge, dans la dernière partie de son livre au sujet de la nouvelle culture du métier. S'il voit le journalisme participatif comme une ruse pour fidéliser le lecteur, il le compare aussi au management, qui dès les années 70 a favorisé la créativité et la capacité d'initiative des employés pour en augmenter la productivité. De la même manière, le journalisme web exploite aujourd'hui le potentiel productif de ses lecteurs par la "servuction", en les mettant littéralement au travail dans les forums, blogs et autres espaces circonscrits. Alors oui, l'information web est encore largement gratuite, mais l'internaute- consommateur participe à la production de l'information, et à sa diffusion, en s'équipant de matériel informatique et web. Ce nouveau type de client de l'information s'insère dans une ère nouvelle, celle des médias personnalisés, selon la logique de la demande. Si l'on a critiqué la faiblesse d'une certaine culture de masse, on peut se demander ce qu'il adviendra de son appauvrissement aux champs d'intérêts d'un individu. Mais le journalisme web n'est pas le seul à bénéficier de ces constats. Il est comme un miroir des tendances, un«laboratoire» pour le renouvellement des pratiques et représentations journalistiques. Il ne fait qu'accélérer un processus de transformation du journalisme, déjà amorcé par ailleurs.

\section{AUTEURS}

\section{AMANDINE DEGAND}

ARC-ORM, université catholique de Louvain amandine.degand@uclouvain.be 